\title{
Neuroimaging and Neurophysiology Studies in Carriers of Cree Leukoencephalopathy
}

\author{
R.J.Huntsman, E.G. Lemire, C.L. Voll, S. Wiebe, N.J. Lowry
}

Can J Neurol Sci. 2011; 38: 347-348

Cree Leukoencephalopathy (CLE) is a rapidly progressive and invariably fatal neurodegenerative disorder that affects children of Cree and Chippewayan descent. It is also referred to as Cree Leukodystrophy. It is an autosomal recessive condition with patients being homozygous for a mutation in the gene that encodes the $\varepsilon$ subunit of the Eukaryotic Initiation Factor 2B (EIF2B5). Cree Leukoencephalopathy is allelic to Vanishing White Matter Disease, one of the most prevalent inherited white matter disorders. ${ }^{1,2}$

Affected children become symptomatic in the first year of life usually following a mild viral infection. They undergo a rapid neurological decline with progressive spasticity and blindness leading to decerebrate posturing, obtundation and death. Magnetic resonance imaging (MRI) shows extensive, symmetric white matter loss in the brainstem and cerebrum, with involvement of the subcortical U-fibres. ${ }^{3}$ Magnetic resonance spectroscopy (MRS) demonstrates diminished $\mathrm{N}$-acetylaspartate (NAA), elevated choline and the presence of lactate peaks in keeping with the breakdown of myelin and physiological stress. ${ }^{1}$ Affected children also have a peripheral neuropathy involving the large myelinated sensory fibres similar to that seen in metachromatic leukodystrophy. ${ }^{1}$

Recently, a two-year-old male sibling of two children who died of CLE was investigated for recurrent seizures arising from the right hemisphere. Genetic studies showed that he was a heterozygous carrier for CLE. His MRI was normal, however MRS with multivoxel Point Resolved Spectroscopy (PRESS) acquisition (TRE/TE $=3000 / 144 \mathrm{msec}$ ) obtained at the level of the corona radiata showed many voxels within the right frontal white matter to have diminished NAA/creatine and elevated choline/creatine ratios. Lactate peaks were not identified.

Manifesting heterozygotes have been reported in various autosomal recessive neurological conditions such as Giant axonal neuropathy, McCardle disease and carnitine palmitoyltransferase deficiency type II. In general, they have much milder symptoms when compared to homozygotes..$^{4-6}$ The presence of an abnormal MRS in our patient raised the possibility that manifesting heterozygotes could occur among CLE carriers. The presence of detectable changes in the nervous systems of EIF2B mutation carriers has not been previously assessed in a systematic manner. We decided to test this hypothesis by inviting known adult CLE carriers to undergo a formal neurological assessment, nerve conduction studies and MRI/MRS.

\section{Materials ANd Methods}

Approval for this study was obtained from the University of Saskatchewan Biomedical Research Ethics Board. Six parents of children who died from CLE and who were genetically confirmed carriers were identified in our institution's genetic database. Four of the parents agreed to participate in the study.

The participants underwent a neurological assessment including a detailed history and full neurological examination. Motor and sensory nerve conduction studies were obtained using a Viking Nikolai EMG machine. Filter settings were set at standard motor (2hz -10khz) and sensory (20-2khz) settings. Motor nerves tested were Peroneal, Tibial, Median and Ulnar on the right side. Sensory nerves tested were Ulnar Digit V, Median Digit II, Peroneal and Sural on the right side. Skin temperature on tested limbs ranged from 32 to 34.8 degrees Celsius.

Brain MRI and MRS were obtained using a Siemens Avanto 1.5 Tesla scanner. Sequences obtained in all patients were sagittal and axial T1, axial T2 and FLAIR, Diffusion Weighted Imaging and Susceptibility Weighted Imaging. Multivoxel PRESS MRS (TRE/TE 1500/135ms) through the axial plane within the periventricular white matter and basal ganglia bilaterally.

\section{RESUlts}

Four adult participants (one male, three female) ranging in age from age 38 to 41 years participated in this study. All were known carriers for the G584A mutation in the EIF2B5 gene on chromosome 3q27. On neurological review of systems, all three females suffered from migraine headache. None of the participants had any history of neurological deterioration following infection or trauma. No other neurological symptoms were elicited. Cranial nerve examination was normal. In the extremities, assessment of tone, strength and tendon reflexes were normal. Plantars were downgoing in all participants. Sensory assessment of light touch, pin prick, vibration and joint position was normal. All participants had normal gaits.

Nerve conduction studies performed in all four participants showed normal motor and sensory responses with values for distal latency, amplitude and conduction velocity falling within the normative values for our institution. ${ }^{7}$

From the Division of Pediatric Neurology (RJH, NJL), Division of Medical Genetics

(EGL), Department of Pediatrics, Division of Neurology (CLV), Department of

Medicine, Department of Medical Imaging (SW), University of Saskatchewan,

Saskatoon, Saskatchewan, Canada.

Received May 12, 2010. Final Revisions Submitted October 14, 2010. Correspondence to: Richard J. Huntsman, Division of Pediatric Neurology,

Department of Pediatrics, University of Saskatchewan, Royal University Hospital,

103 Hospital Drive, Saskatoon, Saskatchewan, S7N 0W8, Canada. 
Magnetic resonance imaging was normal in all four participants with the exception of non-specific mineralization/ calcification seen within the basal ganglia and dentate nuclei of the cerebellar hemispheres on susceptibility weighted imaging in one of the female participants. These changes were felt to be due to normal physiological changes or an endocrine disorder. None of the imaging studies showed any white matter changes in keeping with a leukodystrophy. Likewise, the MRS studies in all four participants revealed normal creatine, choline and NAA ratios and no identifiable lactate peaks were seen.

\section{Discussion}

Cree Leukoencephalopathy is a devastating neurological disease that affects children of Cree and Chippewayan descent. It is one of the most severe variants of Vanishing White Matter Disease and is due to a genetic mutation within the $\varepsilon$ subunit of the Eukaryotic Initiation Factor (EIF2B) encoded on chromosome 3 q27.

Eukaryotic Initiation Factor $2 \mathrm{~B}$ plays a major role in the regulation of protein production during times of physiological stress. During times of physiological stress such as fever, cellular protein production decreases to prevent the formation of denatured protein within the endoplasmic reticulum which results in cellular dysfunction. Mutations within the EIF2B complex impair the ability of the cell to decrease protein production even when it is detrimental to the cell. ${ }^{8-10}$ Recent research has demonstrated an excessive activation of the unfolded protein response as another possible mechanism for cellular dysfunction caused by mutations in the EIF2B complex. In the setting of an EIF2B mutation, the excessive activation of the unfolded protein response results in a marked pro-apoptotic state within the cell even in the absence of physiological stress. ${ }^{8-10}$

Although laboratory studies have shown that at the cellular level heterozygote carriers of EIF2B mutations have normal EIF2B activity, to date no studies have been performed in a systematic manner to assess carriers for any effects within their nervous systems as a result of a heterozygous mutation within the EIF2B complex. ${ }^{8}$ Carriers for the G584A mutation that causes CLE were felt to be an ideal population to study as the mutation in the homozygous state causes such a severe phenotype, making the presence of manifesting heterozygotes more likely than in the milder phenotypes of vanishing white matter disease.

In conclusion, we were not able to demonstrate any abnormalities on detailed neurological assessment attributable to the carrier state for a mutation within the EIF2B complex. The abnormalities detected on MRS in the child with frontal lobe seizures likely reflected the presence of an epileptogenic focus within this region and were not related to his being a carrier for Cree Leukodystrophy. ${ }^{11}$

\section{ACKNOWLEDGEMENTS}

Financial support for this study was provided by a research grant from the Royal University Hopsital Foundation while operational support was provided by the Saskatoon Health Region. The authors thank the participants for kindly giving their time to take part in this study and Doris Neumeyer R.N. for her assistance in coordinating the studies performed.

\section{REFERENCES}

1. Huntsman RJ, Seshia S, Lowry N, Lemire EG, Harder SL. Peripheral neuropathy in a child with Cree Leukodystrophy. J Child Neurol. 2007;22:766-8.

2. Fogli A, Wong K, Eymard-Pierre A, et al. Cree leukoencephalopathy and $\mathrm{CACH} / \mathrm{VWM}$ disease are allelic at the EIF2B5 locus. Ann Neurol. 2002;52:506-10.

3. Alorainey IA, Patenaude YG, O'Gorman AM, Black DN, MeagherVilemure K. Cree leukoencephalopathy: neuroimaging finding. Radiology. 1999;213:400-6.

4. Houlden H, Groves M, Miedzybrodzka Z, et al. New mutations, genotype phenotype studies and manifesting carriers in giant axonal neuropathy. J Neurol Neurosurg Psychiatry. 2007;78: 1267-70.

5. Manfredi G, Silvestri G, Serividei S, et al. Manifesting heterozygotes in McArdle's disease: clinical, morphological and biochemical studies in a family. J Neurol Sci. 1993;115(1):91-4.

6. Rafay M, Murphy EG, McGarry JD, Kaufmann P, DiMauro S, Tein I. Clinical and biochemical heterogeneity in an Italian family with CPT II deficiency due to Ser 113 Leu mutation. Can J Neurol Sci. 2005;32:316-20.

7. Preston DC, Shapiro BE. Electromyography and neuromuscular disorders: clinical-electrophysiological correlations. 2nd ed. Philadelphia: Elsevier; 2005.

8. Schiffman R, Elroy-Stein O. Childhood ataxia with CNS hypomyelination/vanishing white matter disease - a common leukodystrophy caused by abnormal control of protein synthesis. Mol Genet Metab. 2006;88:7-15.

9. van der Voorn JP, van Kollenberg B, Bertrand G, et al. The unfolded protein response in vanishing white matter disease. J Neuropathol Exp Neurol. 2005;64(9):770-5.

10. Van Kollenburg B, van Dijk J, Garbern J, et al. Glia-specific activation of all pathways of the unfolded protein response in vanishing white matter disease. J Neuropathol Exp Neurol. 2006; 65(7):707-15.

11. Hajek M, Dezortova M, Krsek P. 1H MR spectroscopy in epilepsy. Eur J Radiol. 2008;67:258-67. 